Supporting Information

\title{
Comparison of Modular PEG Incorporation Strategies for Stabilization of Peptide- SiRNA Nanocomplexes
}

Justin H. Lo, ${ }^{\dagger,+, \#}$ Ester J. Kwon, ${ }^{\dagger, \#}$ Angela Q. Zhang, ${ }^{\dagger,+}$ Preeti Singhal, ${ }^{\dagger}$ and Sangeeta N. Bhatia $^{*, \dagger, t, s, \|, \perp}$

$\dagger$ Koch Institute for Integrative Cancer Research, MIT, 500 Main Street, Cambridge, Massachusetts 02139, United States

+ Harvard-MIT Division of Health Sciences and Technology and $\S$ Department of Electrical Engineering and Computer Science, MIT, 77 Massachusetts Avenue, Cambridge, Massachusetts 02139, United States

II Department of Medicine, Brigham and Women's Hospital, 75 Francis Street, Boston, Massachusetts 02115 , United States

$\perp$ Howard Hughes Medical Institute, 4000 Jones Bridge Road, Chevy Chase, Maryland 20815, United States

\# These authors contributed equally to this manuscript

* Corresponding author
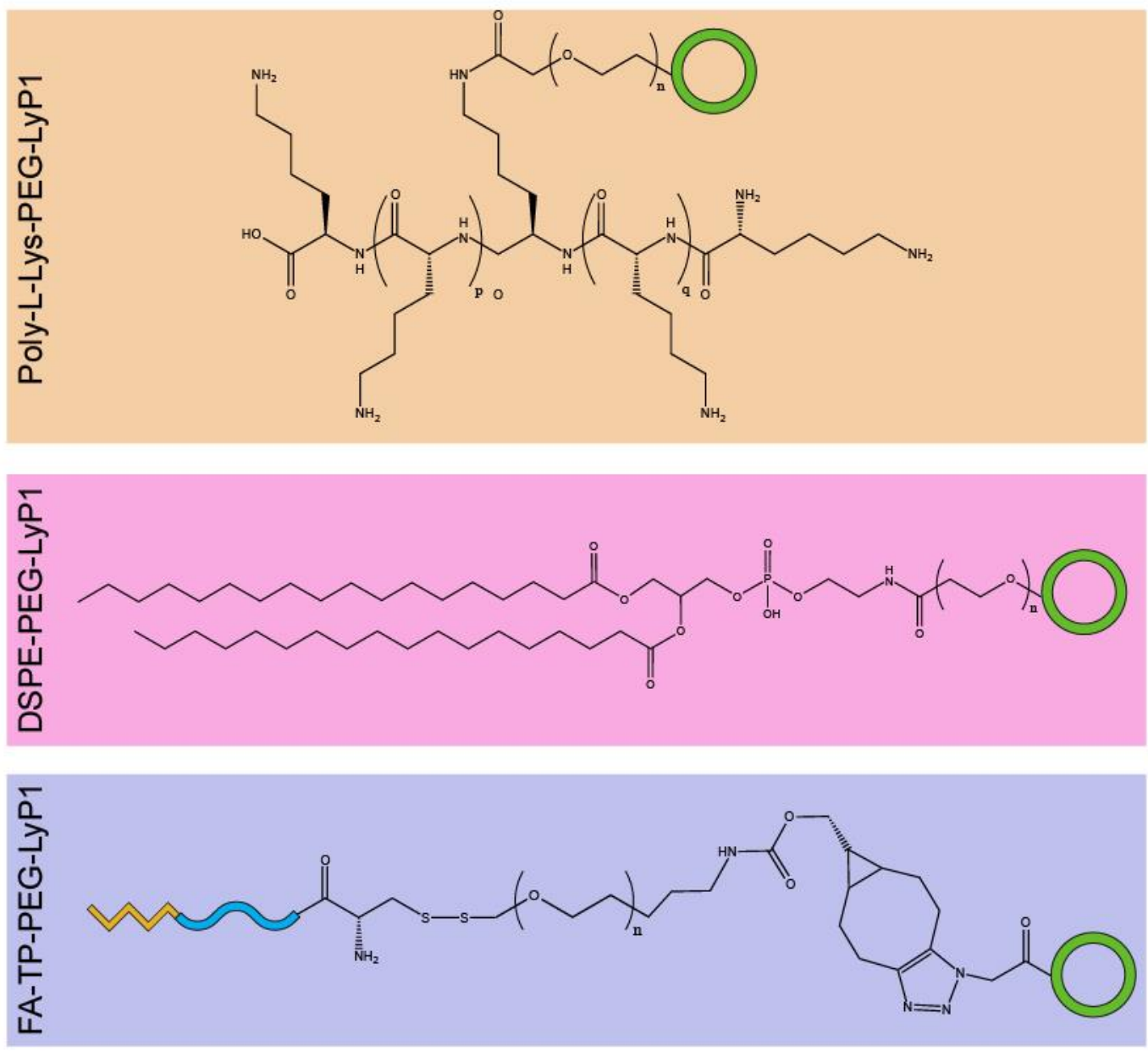

Figure S1. Expected chemical structures of the modular PEG components. The green circle indicates the position of the cyclic LyP-1 peptide, if added. 

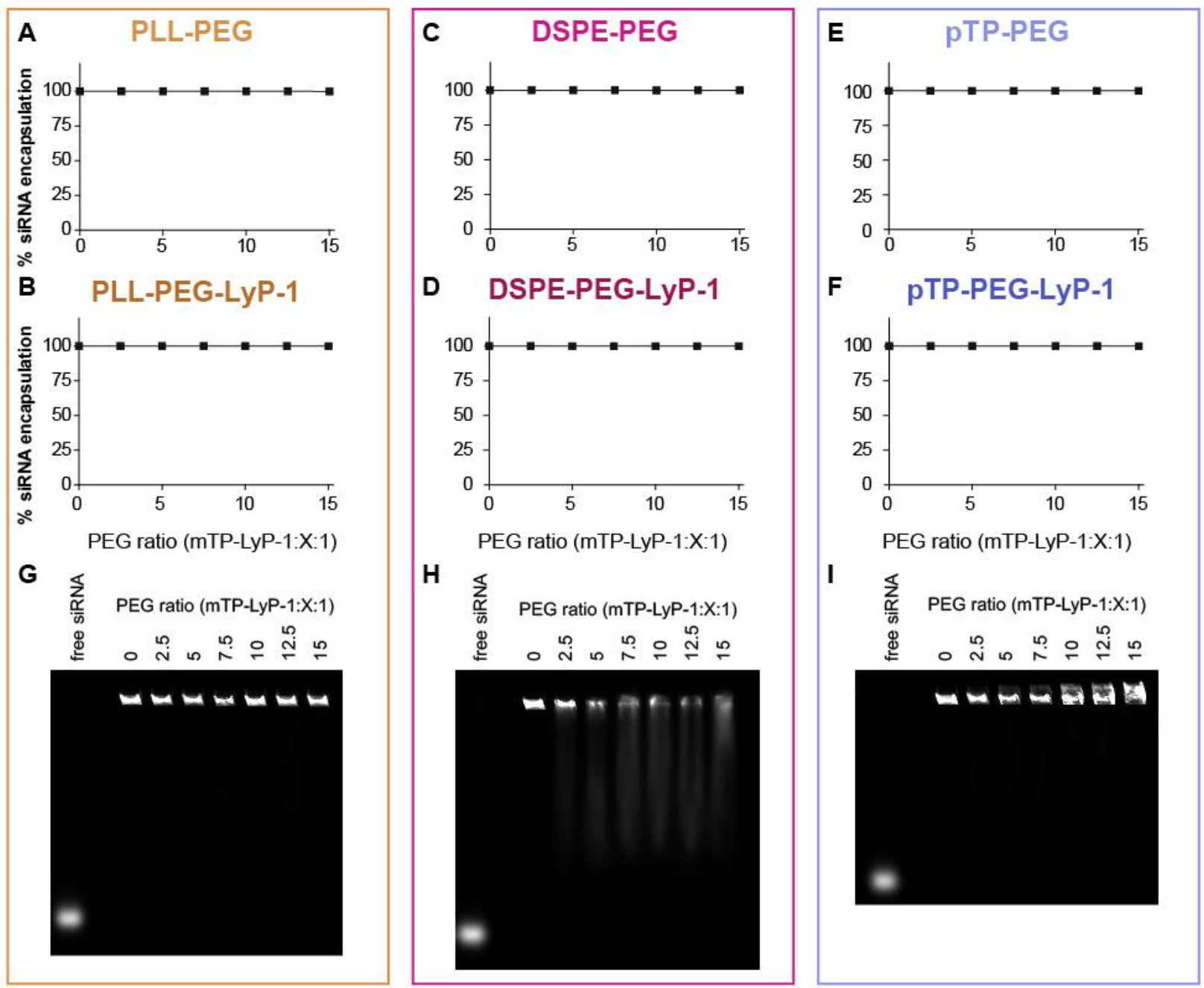

Figure S2. siRNA cargo encapsulation efficiency in PEG TPNs (A-F) Percentage of free siRNA encapsulated by PEG-containing TPNs, as quantified from an electrophoresis mobility shift assay in agarose gels. Scans of agarose gels used for analysis are shown for PLL-PEG-LyP-1 (G), DSPE-PEGLyP-1 (H), and pTP-PEG-LyP-1 (I). While very small nanocomplexes could migrate in the gel, with direction dependent on net charge, in no cases was there a free siRNA band that runs with the free siRNA control in the left lane of each gel. 
pTP-PEG TPN

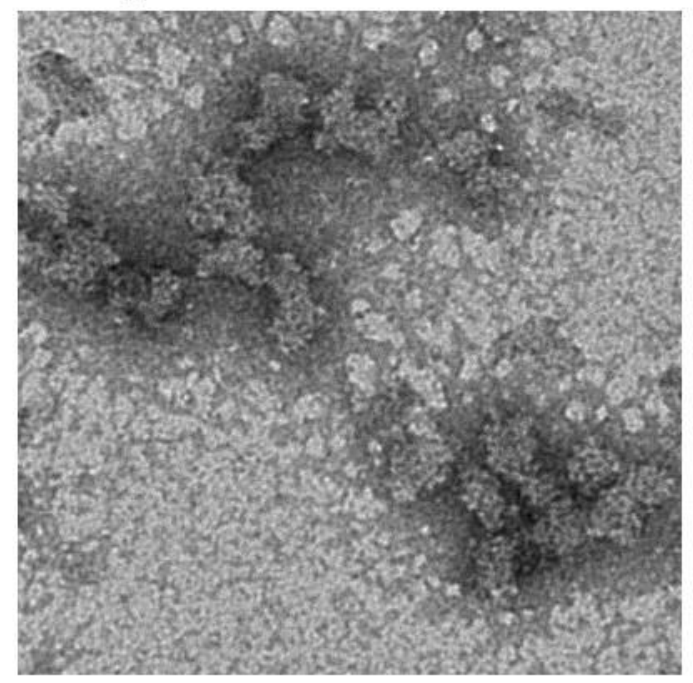

DSPE-PEG TPN

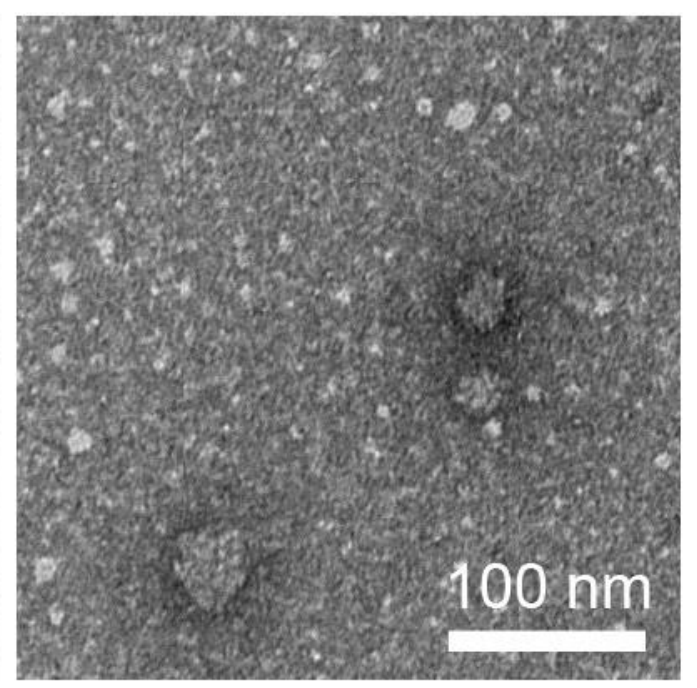

Figure S3. TEM micrographs. Negatively-stained TEMs of 15:15:1 pTP-PEG TPNs (left) and 15:15:1 DSPE-PEG TPNs (right) formed in 1x PBS. 


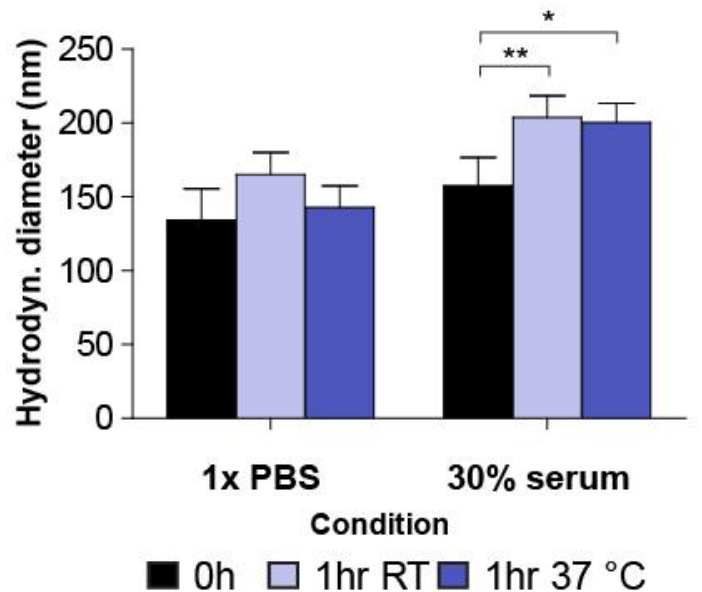

Figure S4. Serum stability of PEG TPNs. Diameters of 15:15:1 mTP-LyP-1:pTP-PEG-LyP-1:siRNA particles in isotonic buffer or $30 \%$ exosome-free FBS at room temperature or physiologic temperature. Statistical significance evaluated by two-way ANOVA with Bonferroni post-test. ${ }^{*}$ : $p<0.05,{ }^{* *}$ : $p<0.01$. 
A

DSPE-PEG

poly-glutamic acid:siRNA ratio

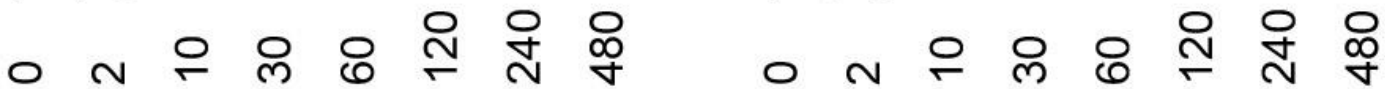

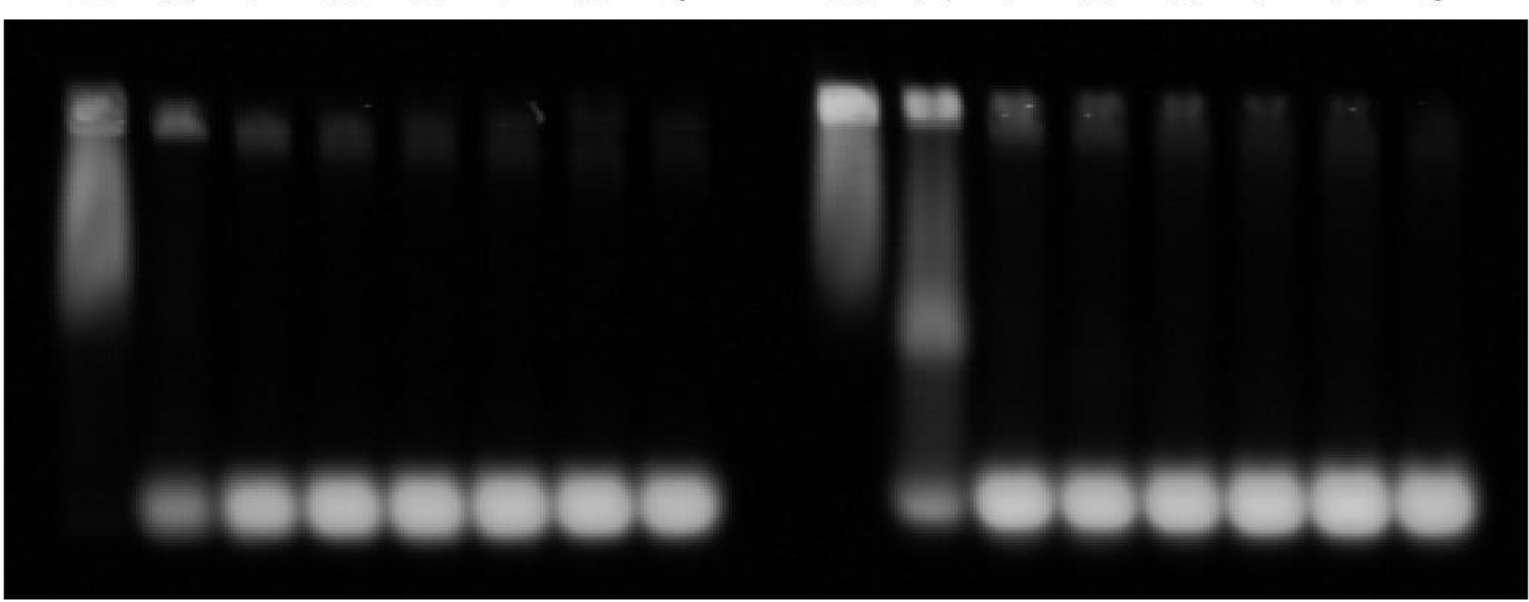

Figure S5. Polyanion exchange gel electrophoresis. PEG TPNs formed with (A) DSPE-PEG or (B) pTP-PEG were mixed with increasing molar ratios of poly-glutamic acid to siRNA (with the concentration of the particles held constant). Appearance of the free siRNA band (bottom) and loss of siRNA retained in the loading wells (top) corresponds with the degree of particle disruption and displacement of siRNA by poly-glutamic acid. 


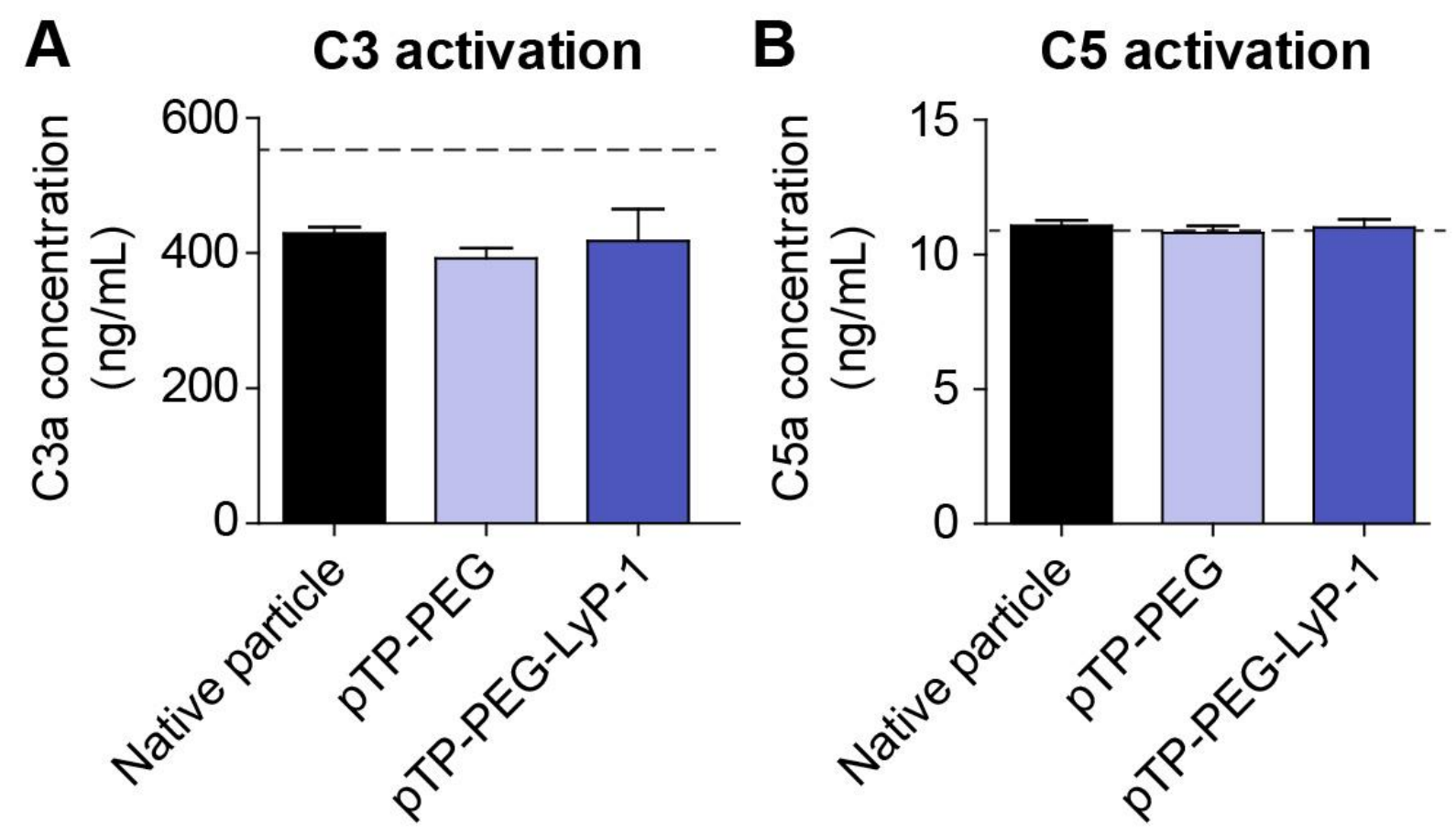

Figure S6. Complement activation. Levels of (A) C3a or C3a-desArg and (B) C5a or C5a-desArg after incubation of non-PEGylated TPNs (15:1 peptide:siRNA ratio), pTP-PEG TPNs (15:15:1), or pTP-PEGLyP-1 particles (15:15:1) with human plasma. 